RESEARCH NOTE

\section{Structure of the Knob Protein Gene of the Saimiri Monkey-adapted Palo Alto Strain of Plasmodium falciparum}

\section{Wanda Chalvet, Jean-François Pouliquen*, Thierry Fandeur/ ${ }^{+}$}

\author{
Laboratoire de Parasitologie Moléculaire \\ *Laboratoire des Rétrovirus, Institut Pasteur de la \\ Guyane, BP 6010, 97306 Cayenne Cedex, \\ French Guiana
}

Key words: Plasmodium falciparum - histidine-rich protein - Palo Alto parasites - squirrel monkey - strain infectivity

The histidine-rich protein is the major component of the knob-like protrusions detected on the membrane of both human and monkey erythrocytes infected with Plasmodium falciparum (SA Luse \& LH Miller 1971 Am J Trop Med Hyg 20: 655660, M Hommel et al. 1982 Parasite Immunol 4: 409-419, F Ardeshir et al. 1987 EMBO 6: 14211427). The knobs on the red blood cell surface are generally associated with strain pathogenicity and virulence. Isolates collected from naturally infected patients are consistently of knobby phenotype (Hommel et al. loc. cit.) and knobless lines were reported to be less virulent in monkey models ( $\mathrm{S}$ Langreth \& E Peterson 1985 Inf Immun 47: 760766, T Fandeur et al. 1992 Inf Immun 60: 13901396).

Experimental infection of the squirrel monkey, Saimiri sciureus, with $P$. falciparum is one of these well-established monkey models for human malaria. Recently, we examined several monkeyadapted and culture-derived $P$. falciparum strains for their ability to propagate in splenectomized Saimiri monkeys. Consistent with the above, we found a correlation between allelic diversity at the HRPI locus and the course of blood stage infection (T Fandeur et al. 1996 Exp Parasitol 84: 115). A particular allele of the HRPI gene was found

\footnotetext{
${ }^{+}$Corresponding author. Fax: +5-94-31.8083

Received 20 September 1997

Accepted 8 January 1998
}

only in strains presenting a FUPSP genotype and growing to high parasitic density. However, strains possessing a wild-type HRPI gene (FUPCB, FVO, Geneva, Sal I, Honduras and FCH4) or lacking the HRPI sequences (FUPCP), produced no to lowdensity infections in splenectomized Saimiri, thereby suggesting that this particular HRPI allele is fundamental for the P. falciparum infection of $S$. sciureus erythrocytes. We now report the partial sequence of the HRPI allele which was associated with rapid developing infection in the splenectomized Saimiri.

The HRPI gene shown in Fig. 1a includes a region encoding a histidine-rich portion in the second exon, and two blocks of repeated sequences. The gene in FUPSP genomic DNA was amplified with primers P1 (CCGGGATCCATGAAAAGTTT TAAGAACAA, positions 629 to 657) and P2 (TGAATTCCCTGCACCATGGGGTGGG, positions 1597 to 1621 ), and with primers P3 (CCGGATCCCACCCCATGGTGCAGGC, positions 1590 to 1614) and P4 (AGAATTCCATT GTCCTTTATTTGTTGCGGC, positions 2216 to 2245), as deduced from the HRPI sequence in FCR3 parasites (LG Pologe et al. 1987 Proc Nat Acad Sci, USA 84: 7139-7143). The artificial BamHI or EcorI sites introduced at the 5' end of the primers are underlined. Priming the HRPI sequences with oligonucleotides P1-P2 amplified a 1430 bp PCR-product (Fig. 1B, lane 1), corresponding to a wild-type HRPI sequence, whereas the $530 \mathrm{bp}$ fragment produced by using primers P3-P4 (Fig. 1B, lane 3) is shorter than expected from the previously published FCR3 and NF7 sequences (Pologe et al. loc. cit., T Triglia et al. 1987 EMBO 6: 1413-1419). Indeed, amplification of an intact $640 \mathrm{bp}$ fragment was uniformly observed in FUPCB, FVO, Geneva, Sal I, Honduras and FCH4 parasites, all producing low-density and self-cured infections in splenectomized monkeys (Fandeur et al. loc. cit.). The fragments labeled A and B in Fig. 1 were further characterized by cloning and sequencing. The PCR-products were restricted with BamHI-EcorI, excised from the gel, purified by Geneclean $\mathrm{II}^{\mathrm{R}}$, and finally cloned into Bam HI-EcorI digested M13 vectors. Digestion of the $1430 \mathrm{bp}$ fragment (Fig. 1B, lane 1) produced two restriction fragments of about 870 and $570 \mathrm{bp}$ respectively (Fig. 1B, lane 2). The BamHI restriction fragment located in the $5^{\prime}$ region and containing the intron was not further studied.

The nucleotide sequences obtained of several independent bacteriophages carrying HRPI fragments from FUPSP strain were determined (Fig. 2 ). The sequences of the PCR-amplified regions $\mathrm{A}$ and $\mathrm{B}$ of the gene (Fig. 1A) were joined end to 


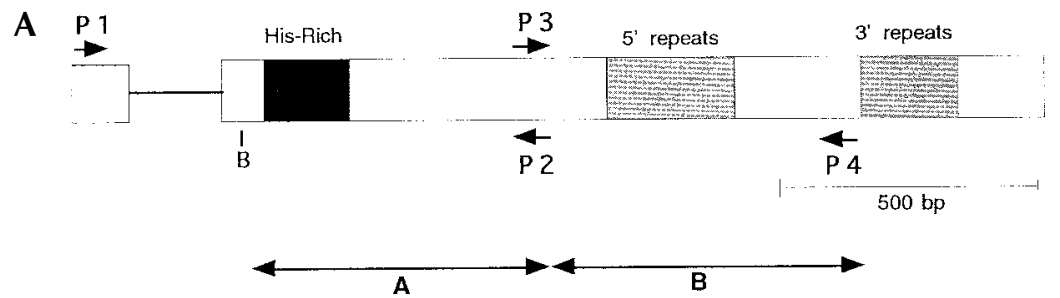

B

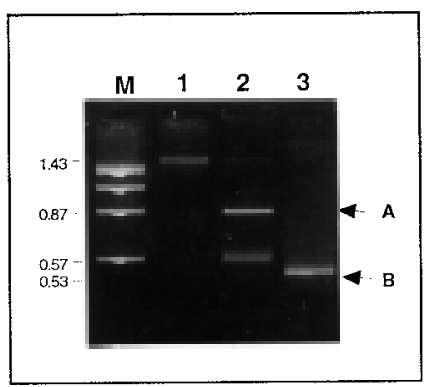

Fig. 1: A - Structure of the HRPI gene of Plasmodium falciparum. Boxed areas indicate the coding regions. 5' and 3' repeats, and the histidine-rich regions are shaded. The positions of the oligonucleotides P1-P4 used for the PCR reactions, and the positions of PCR-fragments A and B sequenced after transfer to M13 are indicated. The BamHI (B) restriction site near the N-terminal histidine-rich domain of the gene is shown. Oligonucleotides P3 and P4 are identical to those previously labeled HRPI A and D in an other study (T Fandeur et al. 1996 Exp Parasitol 84: 1-15), B - PCR-products generated with oligonucleotides P1-P2 (lane 1) and P3-P4 (fragment B, lane 3) by using FUPSP DNA as template. The $1.4 \mathrm{~kb}$ PCR product amplified by using primers P1 and P2, and digested with BamHI-EcoRI (lane 2).

end, and aligned to those previously published for the FCR3 and NF7 strains. The HRPI gene of FUPSP was closely related to that of FCR3 because both sequences lacked the 13 bases at positions 70-75, 102-107 and 1271 in the consensus sequence. Single base deletions at positions 848 , 850,859 and 1192 were detected only in the NF7 sequence. FUPSP and FCR3 sequences differed from each other by (i) seven point mutations substituting Q, D, L, G, K, L, E in FCR-3 for $\mathrm{H}, \mathrm{N}$, P, D, E, S, Q in FUPSP at positions 90, 99, 254, $755,1267,1287$ and 1348, respectively; and by (ii) a large deletion of $81 \mathrm{bp}$ (1111 to 1192) in the FUPSP sequence.

Although the HRPI gene is generally well conserved in these isolates, the few base substitutions and deletions have consequences on the encoded proteins. The deduced amino acid sequences differed mostly in the region between from amino acid residues 370 and 440 (Fig. 3). A frame shift of NF7 occurs twice. The deletion of an A at position 1192 in the NF7 nucleotide sequence modifies the reading frame from codon 398 , it was then corrected at position 424. No similar change in the reading frame was observed for the FUPSP sequence despite a large deletion removing the fourth and fifth 5' repeats. Comparison of the predicted secondary a-helix and b-sheet structures of the protein in NF7, FUPSP and FCR3 indicated that such changes result in altering the conformation of this region (data not shown), in other respect described as highly immunogenic (MA Rashid et al. 1990 Mol Biochem Parasitol 38: 49-56), and also identified as being the functional domain of the knob protein (A Kilejian et al. $1991 \mathrm{Mol}$ Biochem Parasitol 44: 175-182). These modifications are expected to have some effect on the antigenic and functional properties of HRPI in the context of FUPSP parasites.

A general problem encountered when studying parasite virulence is to define what virulence means. There is no single definition both satisfactory and relevant for all situations studied. During infection of the splenectomized monkeys with the various $P$. falciparum strains, we did not detect any sign of severe disease, except for hyperparasitemia. In addition, trophozoite and schizonte stages do circulate in splenectomized animals, indicating that in this host low-grade parasitemias do not rely to extensive parasite sequestration to capillary endothelium. Consequently, we considered appropriate to assess strain virulence by monitoring parasitemia. Based on this criterion, a correlation was established between 


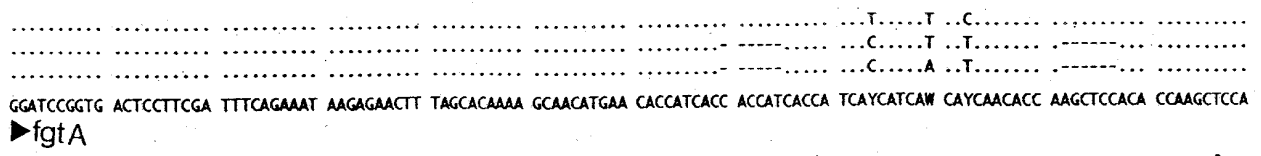

fgt $A$

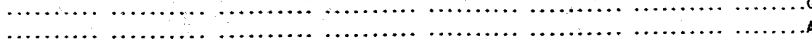

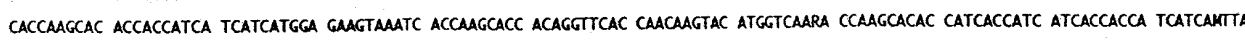

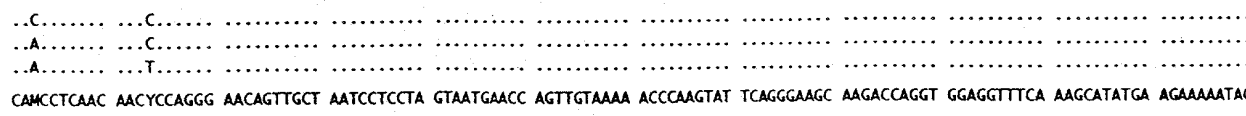

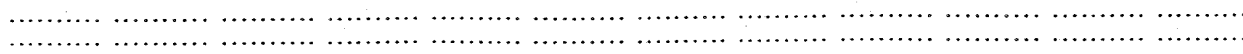

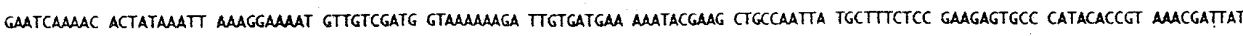

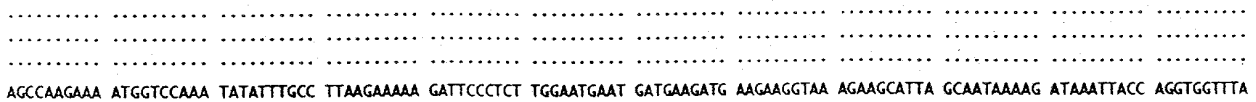

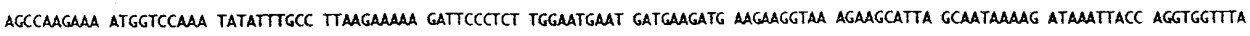
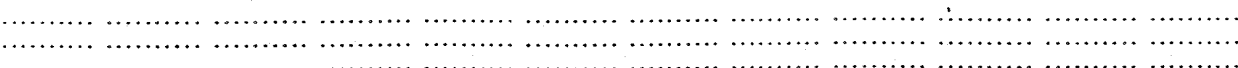

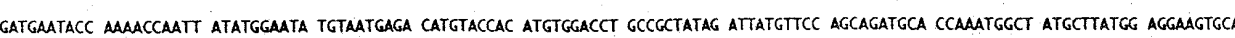

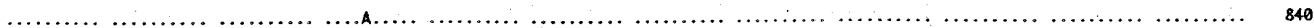

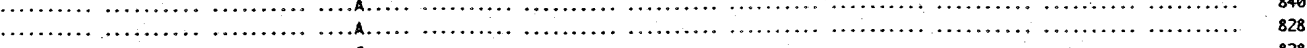

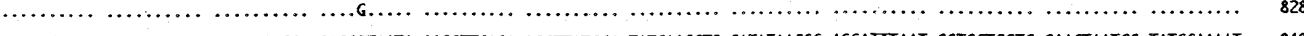
CACGATGGT CTCACGGTAA TITAAGAGGA CACGRTAATA AAGGTTCAGA AGGTTATGGA TATGAAGCTC CATATAACCC AGGATTTAAT GGTGCTCCTG GAAGTAATGG TATGCAAAT

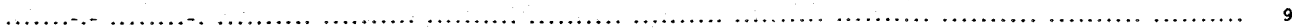

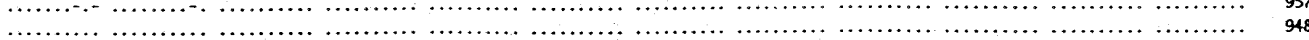

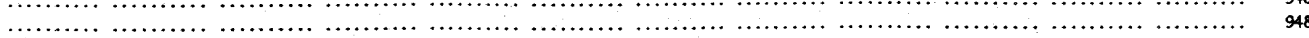
TATGTCCCAC CCCATGGTGC AGGCTATTCA GCTCCATACG GAGTTCCACA TGGTGCAGCC CATGGTTCAA GATATAGTTC ATTCAGTTCC GTAAATAGAT ATGGAaAaCA CGGTGATGAA 96O $D$ fgtB

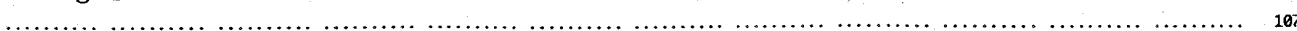

Fig. 2: partial nucleotide sequence of the HRPI gene from the FUPSP strain of Plasmodium falciparum adapted to the Saimiri monkey, and comparison to those reported previously for the FCR3 and NF7 strains. The sequences were aligned using the GeneWorks program (Intelligenetics). The consensus sequence is shown together with differences between the sequences; identities are shown as ( ..... ) and deletions as ( ----- ). Unless specified otherwise, the positions of the bases or amino acids given in the text are based on the numbering of this consensus sequence. This sequence has been submitted to EMBL Nucleotide Sequence Database with the accession number Y10828. The positions of PCR fragments A and B shown in Fig. 1 are indicated. 
NF7

FUPSP

FCR -3

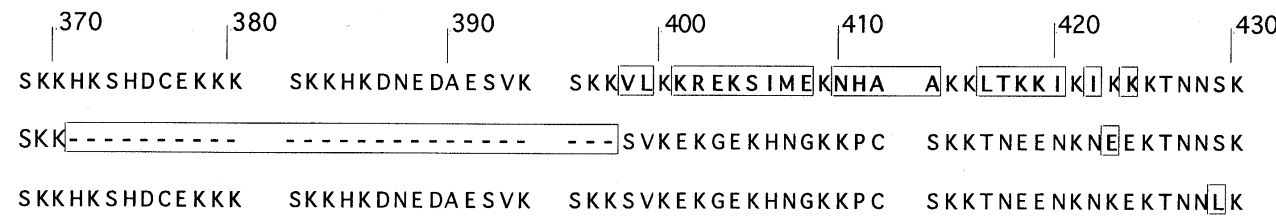

Fig. 3: alignment of predicted amino acid sequences extending from residues 370 to 430 in strains NF7, FUPSP, and FCR3. Unique residues, stretches of sequence, and deletions different between the various alleles specificities of the strains are boxed. Amino acid positions are deduced from the consensus nucleotide sequence shown in Fig. 2.

allelic diversity at the locus encoding the knob protein and the severity of blood infections.

The positive selection in splenectomized monkeys for parasites expressing a particular form of the knob protein is puzzling. By analogy to in vitro observations (JG Culvenor et al. 1987 Exp Parasitol 63: 58-67), we speculate that adaptation to Saimiri, usually associated with increased growth rate, might involve selection of a parasitic strain with this particular allelic form of the HRPI gene. However, we do not understand at present why there is positive selection for these parasites. The function of HRPI remains unknown, yet some evidence has been presented for the knob protein being a malarial parasite analogue of red cell protein 4.1 (Kilejian et al. loc.cit.). This suggests a mechanism whereby HRPI could modify the host cell membrane and directly interfere with parasite development within the infected erythrocyte. The diversity found at the HRPI locus might therefore reflect spectrin diversity of the red blood cell as both proteins associate with the erythrocyte membrane cytoskeleton to form knob complexes.

Acknowledgments: to Bernard Bonnemains for his assistance in managing the monkeys and to Cécile Le Scanf for fruitful discussions. 